\title{
El establecimiento permanente de seguros: análisis crítico de su aplicación desde la perspectiva tributaria colombiana*
}

\section{The Permanent Establishment of Insurance: Critical Analysis of its Application from the Colombian Tax Perspective}

\author{
Omar Sebastián Cabrera Cabrera ${ }^{1}$
}

\section{Sumario}

Introducción. I. Aspectos básicos del EP de seguros. A. Un sujeto calificado. B. Una actividad particular de seguros salvo reaseguros. C. Exclusión de agentes independientes. II. El EP de seguros en el Derecho doméstico colombiano. III. El EP de seguros en la red de tratados tributarios de Colombia. IV. Aplicación del EP de seguros en Colombia. A. La intermediación de seguros en el Derecho colombiano. B. Falta de norma doméstica del EP de seguros. C. Requisitos para la existencia de un agente de seguros desde la óptica doméstica. Conclusiones. Bibliografía.

\section{Resumen}

Los convenios para evitar la doble imposición prevén diferentes categorías de establecimientos permanentes, dependiendo de su finalidad y naturaleza. De esta manera, la plantilla de la Naciones Unidas señala una hipótesis especial denominada establecimiento permanente de seguros, la cual no es un elemento típico en el Modelo genérico de la Organisation for Economic Co-operation and Development, OCDE (OECD, en inglés). Sin embargo, en la redacción actual se excluyen del espectro de este precepto los agentes de carácter independiente, lo cual no ha dejado de generar críticas por parte de

* DOI: https://doi.org/10.18601/16926722.n11.09

${ }^{1}$ Abogado con profundización en Derecho Tributario y especialista en Derecho Económico Internacional, de la Universidad Externado de Colombia. Especialista y magíster en Tributación, de la Universidad de los Andes. Ha sido docente asistente de la intensificación en Derecho Tributario Internacional e investigador del Centro de Estudios Fiscales Externadista e investigador del Instituto Colombiano de Derecho Tributario. También cursó Diplomado en Impuestos en la Facultad de Contaduría Pública de la Universidad Externado de Colombia. Consultor tributario en Price Water House Coopers, Colombia. Correo-e: omarcabreracabrera@gmail.com 
los países importadores de capital. De otro lado, la regulación colombiana del establecimiento permanente omitió la inclusión del establecimiento permanente de seguros, lo cual ha causado incompatibilidad con ciertos tratados tributarios suscritos por Colombia.

\section{Palabras clave}

Establecimiento permanente, Establecimiento permanente de seguros, Agentes de seguros, Tributación de los beneficios empresariales.

\section{Abstract}

Double Taxation Conventions provide several categories of permanent establishments depending on their purpose and nature. In this sense, the United Nations tax convention set forth a special hypothesis so-called the permanent establishment of insurance, which is not a typical element in the Organisation for Economic Co-operation and Development, OECD (OCDE, in spanish) Model. However, the scope of the current wording excludes the agents of an independent status, which has generated criticism from capital importing countries. On the other hand, the Colombian regulation of the permanent establishment lacks the inclusion of the permanent establishment of insurance generating incompatibilities with certain tax treaties signed by Colombia.

\section{Keywords}

Permanent Establishment, Insurance Permanent Establishment, Assurance Brokers, Taxation of Business Profits.

\section{Introducción}

El concepto del establecimiento permanente (EP) previsto en los convenios para evitar la doble imposición (CDI) pretende eliminar la doble tributación jurídica internacional de las denominadas rentas activas (señaladas en el convenio como rentas o beneficios empresariales o business profits) que un residente fiscal de un Estado obtiene en el otro Estado contratante o en cualquier otro país o territorio (Cabrera Cabrera, 2017), dada la naturaleza de multilateralidad de la disposición ${ }^{2}$.

Dicho esto, cuando un residente percibe una renta activa en el otro Estado signatario sin que medie la presencia de un EP, existirá tributación exclusiva en el país de la residencia. Empero, en el evento en que la actividad comercial se desarrolle a través de un EP, el Estado de la fuente podrá gravar la actividad mercantil, solo que de manera limitada a aquellos ingresos o beneficios que le sean imputables al EP según los lineamientos y criterios señalados en el informe de OECD Authorized Approach de 2010 (Reimer, 2015).

Por su parte, vale señalar que bajo estos acuerdos fiscales, el EP no es considerado como persona y, por tanto, es visto como una mera extensión económica del no residente en la fuente (Skaar, 1991). Doctrinalmente hablando, el EP es un umbral que determina la graduación de la vinculación o penetración económica que se tenga en el otro Estado (Goblit y Vaquero, 2011).Así las 
cosas, desde aquella lejana publicación del informe del comité de expertos económicos de la Liga de las Naciones, en 1922, se ha sostenido que la tributación in situ solo debe ser permitida en aquellos casos en los cuales sea notoria y precisa una presencia económica relevante (Vogel, 2011).

Ahora, desde un punto de vista del Derecho tributario doméstico, el criterio o nexo de EP ha sido empleado de manera generalizada en múltiples jurisdicciones tributarias como un instrumento para el sometimiento tributario objetivo, tanto formal como sustancial, de los no residentes fiscales, bien sean personas físicas o jurídicas, cuando desarrollan actividades de negocio de manera permanente y continua en el Estado de la fuente (Sasseville y Skaar, 2009).

Lo consignado en las líneas anteriores permite introducir la repercusión o efecto de transcendencia del EP en el reino del Derecho Tributario Internacional (DTI), tomada esta última disciplina jurídica en su acepción más general ${ }^{3}$, teniendo en cuenta la importancia del Derecho interno como fuente de esta disciplina. Así las cosas, es sencillo comprender que la cuestión de si existe o no un EP se ha convertido, quizás, en una de las preguntas de mayor relevancia tanto para las autoridades tributarias locales como para los contribuyentes (Dunahoo, 2008; Tillinghast, 1993).

Asimismo, es posible evidenciar, tanto en los tratados tributarios como en las normas internas, diferentes tipos o formas de EP en cadencia con su naturaleza intrínseca. Dicho esto, se pueden señalar como ejemplos: el EP regla base (basic rule), de construcción (construction PE), de servicios (services PE), de seguros (insurance PE), los EP fuera de costa u offshore, entre otros. Sentado lo anterior, cada una de estas hipótesis de EP responden a supuestos de hecho especial, dada la particularidad de cada actividad.

Una vez establecido el marco introductorio, es preciso indicar que el objeto principal de análisis del presente texto se concentrará en hacer un análisis crítico del denominado EP de seguros visto desde: i) la óptica de los modelos de convenios tradicionales, ii) el Derecho interno colombiano iii) y su posible aplicación en los distintos tratados tributarios suscritos por Colombia y con plena vigencia.

\section{Aspectos básicos del EP de seguros}

Como primer punto, debe señalarse que el EP de seguros tiene su origen histórico en el Modelo de Convenio de la Organización de las Naciones Unidas (ONU) y, por ende, no se trata de una disposición que se encuentre consagrada en el prototipo básico del Modelo de la Organización para la Cooperación y el Desarrollo Económicos (OCDE).

Lo anterior en la medida en que se refiere a un supuesto especial de EP que procura ampliar el espectro de tributación en la fuente de los beneficios empresariales, finalidad

\footnotetext{
${ }^{3}$ Entendiendo que las fuentes de Derecho Tributario Internacional involucran no solo tratados tributarios sino también normas jurídicas internas.
} 
que está en línea con la teleología misma del Modelo de la ONU, a saber, ser un tratado más garantista para los países en vías de desarrollo cuando estos últimos celebran acuerdos tributarios con países desarrollados o del primer mundo.

Sin embargo, resulta importante poner de presente que los comentarios al Modelo de la OCDE reconocen la posibilidad y la práctica, de alguna manera general, de incluir una provisión de EP de seguros en las negociaciones bilaterales de los Estados miembros de esta afamada organización internacional. A pesar de ello, ha existido bastante resiliencia en incluir formalmente el precepto del EP de seguros en el texto del Modelo OCDE, de tal suerte que el Comentario del artículo 5, párrafo 39 Modelo de la OCDE, dispone lo siguiente:

Conforme a la definición de la expresión "establecimiento permanente", una compañía de seguros de un Estado puede someterse a imposición en el otro Estado por sus operaciones de seguro si tiene un lugar fijo de negocios en el sentido del apartado 1 o si realiza sus actividades mediante una persona en las condiciones del apartado 5. Dado que las agencias de compañías de seguros extranjeras no siempre responden a uno $\mathrm{u}$ otro de estos dos supuestos, puede ocurrir que dichas compañías realicen actividades a gran escala en un Estado sin que se sometan a imposición en ese Estado por los beneficios generados por tales actividades. Para evitar esa situación, varios convenios suscritos por países miembros de la OCDE contienen una disposición conforme a la cual se considera que las compañías de seguros de un Estado tienen un establecimiento permanente en el otro si cobran primas en ese otro Estado por medio de un agente establecido allí -distinto de un agente que ya tenga la condición de establecimiento permanente en virtud del apartado 5- o aseguran riesgos situados en ese territorio por medio de él. La decisión de incluir una disposición de este tipo en un convenio dependerá de la situación jurídica y fáctica existente en los Estados contratantes. Por consiguiente, será frecuente omitir tal disposición. Por ello, no se ha considerado oportuno incluir una disposición de ese tipo en el Modelo de Convenio (destacado fuera de texto).

Una vez sentadas las observaciones anteriores, el artículo 5 (7) del Modelo de plantilla de la ONU consagra:

No obstante las disposiciones anteriores del presente artículo, se considerará que una empresa aseguradora de un Estado contratante tiene, salvo por lo que respecta a los reaseguros, un establecimiento permanente en el otro Estado si recauda primas en el territorio de ese Estado o si asegura contra riesgos situados en él por medio de una persona que no sea un representante independiente al que se aplique el párrafo 7 (Artículo 5 (7) Modelo de la ONU).

A raíz de la lectura detallada del precepto, pueden destacarse los elementos o bloques constitutivos generales para la activación del EP de seguros, los cuales son: a) un sujeto calificado denominado agente, b) una actividad particular de seguros salvo las operaciones de reaseguros y c) la exclusión de agentes independientes.

\section{A. Un sujeto calificado}

En primera medida, se requiere la existencia de una persona en el otro Estado con- 
tratante que tenga la naturaleza de ser un agente, llamado coloquialmente como agente de seguros (insurance agent). Dicho esto, este, quien puede ser una persona natural o jurídica, no requiere tener un lugar fijo de negocios conforme los lineamientos del EP regla base en el territorio donde desarrolla su actividad. Al respecto, señala García Prats (1996) que

(...) La referencia a los agentes, o a la agencia, debe entenderse en sentido comercial amplio, sinónimo de representación comercial, y no en su acepción técnico jurídica, entendiéndose como aquella actuación por la que una persona, el representante o intermediario, actúa en nombre de otra persona (p.184).

Asimismo, tampoco es mandatorio que esta persona se encuentre vinculada laboralmente o tenga relación laboral con la entidad aseguradora del exterior, es decir, que no es necesaria subordinación patronal del agente ni tampoco que este reciba un salario en contraprestación del servicio prestado. Por último, es imperativo que la labor aseguradora desempeñada por el agente se realice a favor de una entidad de seguros legalmente constituida bajo las leyes del otro Estado contratante.

De otro lado, es fundamental hacer hincapié en que solamente podrían accionar un EP de seguros aquellos agentes que no posean una naturaleza de dependencia conforme lo previsto en el numeral 5 del artículo 5 de los Modelos de convenio; esto, a su turno, obliga a una remisión tácita frente a las reglas del denominado EP personal o EP de agencia. Lo precedente en la medida en que una solución distinta generaría que se debieran reunir en el agente determinados elementos que imposibilitarían en grado sumo la configuración del EP de seguros. Así la cosas, puede leerse al respecto el siguiente comentario:

El párrafo 6 de la Convención modelo de las Naciones Unidas, que logra el objetivo mencionado precedentemente, resulta necesario porque en general los agentes de seguros no tienen autoridad para concertar contratos; en consecuencia, no se reunirían los requisitos establecidos en el apartado a) del párrafo 5. Sin embargo, si un agente de seguros es independiente, los beneficios obtenidos por la empresa de seguros atribuibles a las actividades del agente no son gravables en el Estado de la fuente porque se cumplirían las disposiciones del párrafo 7 del artículo 5 y se consideraría que la empresa no tiene un establecimiento permanente (destacado fuera de texto) (Comentarios al artículo 5, párrafo 28 Modelo de la ONU).

En consecuencia y, según la redacción del numeral antes descrito, no es requerido que se cumplan todos los requisitos de dependencia según lo expresado en la regla de la agencia. Esto conlleva a señalar que este agente de seguros no tiene por qué reunir las siguientes cualidades:

i) Poseer la facultad de celebrar contratos en nombre de la empresa de seguros y que dichos contratos le sean vinculantes legalmente;

ii) Ejercer dicha potestad en el otro Estado signatario de manera frecuente y no meramente transitoria o esporádica;

iii) Ser dependiente tanto desde el punto de vista jurídico como económico; 
iv) No debe realizar actividades de índole preparatoria o auxiliar según lo establecido en el artículo 5(4) de las plantillas de los CDI.

\section{B. Una actividad particular de seguros salvo reaseguros}

Frente al segundo bloque, debe hacerse hincapié en que el ámbito del EP de seguros se circunscribe exclusivamente a un determinado segmento de negocio en la industria aseguradora. Así las cosas, aplica solo en los casos del recaudo de primas de seguros, distinta de los reaseguros, y del aseguramiento de riesgos ubicados en el país de la fuente. Como puede evidenciarse, se procura ceñir su aplicación para la actividad aseguradora de manera exclusiva.

\section{Exclusión de agentes independientes}

La regla del EP de seguros es clara al disponer que no es aplicable para aquellos agentes previstos en el numeral 7 del artículo 5 del Modelo de la ONU. En términos concretos, la regla antes referida está intencionada a excluir del espectro del EP de agencia a ciertos agentes por su característica de independientes y su actuar en el curso ordinario de sus negocios; es decir, el criterio de agente independiente es la otra cara de la moneda de la dependencia (Title, 2007).

Sin embargo, el hecho de que el espectro de la norma excluya a los agentes independientes ha generado gran revuelo y fuertes críticas en ciertos países, principalmente en los catalogados como netamente importadores de capital. Lo precedente en el entendido de que es bastante frecuente en la práctica que los agentes dedicados a la venta de seguros tengan una calidad de independientes. $\mathrm{La}$ razón radica en que, según los comentarios al Modelo de la ONU, los factores principales para determinar la dependencia de un agente particular son los siguientes: i) número de mandantes, ii) impartición detallada de órdenes y iii) repartición balanceada de los riesgos.

De esta forma, debe hacerse hincapié en que de manera frecuente los agentes de seguros prestan sus servicios para múltiples entidades aseguradoras sin que exista algún tipo de exclusividad o preferencia frente a una de ellas. También, esa variedad genera que no exista una entidad aseguradora que sea preponderante en el actuar del agente. Esto, a su turno, produce que los agentes de seguros no estén sometidos a instrucciones detalladas en lo que respecta a la forma de realizar su gestión, quedando en un margen de liberalidad importante. Igualmente, debe resaltarse el que a pesar de que los agentes de seguros con frecuencia tienen la obligación principal de ofrecer información a sus mandantes, se trata simplemente de una remisión de información natural en una relación contractual que no genera necesariamente la existencia de un nivel o grado de control importante (Fuentes Hernández, 2014).

Por último, es usual que los agentes de seguros reciban una pequeña parte del negocio en su comisión partiendo de la base de que asumen poco riesgo en el negocio, al ser su actividad meramente de intermediación. De la misma manera, por regla general los contratos de corretaje de seguro no corresponden a una naturaleza intuito personae y, por lo tanto, esto implica que la actividad le reporte cierto riesgo leve al agente (Fuentes Hernández, 2014). 
De otro lado, como se señaló, para predicar la independencia de un agente se requiere que este actúe en el curso ordinario de su negocio. En este sentido, resulta sencillo de comprender que los agentes de seguro cumplen con este umbral en la medida en que lo corriente de su actividad es la intermediación en la venta de seguros, lo cual representa su nicho natural de negocios. Ahora, sobre este punto léase que

Para determinar si ciertas actividades entran o no en el campo normal de actuación del agente, se examinarán las realizadas habitualmente en el ámbito de su profesión -como corredor o agente de ventas, comisionista u otro agente independientey no las demás actividades comerciales que lleve a cabo. Aunque se debe hacer normalmente la comparación con las actividades habituales del agente, podrán utilizarse simultánea o alternativamente otros criterios complementarios, por ejemplo, cuando el conjunto de las actividades del agente se desvíe de las propias de su profesión (Comentarios al artículo 5, párrafo 38.8 Modelo de la OCDE).

Por consiguiente, se ha sostenido que dicha exención de independencia hace nugatorio el propósito del EP de seguros en la medida en que existe una dificultad palpable a la hora de diferenciar si un agente de seguros es o no independiente (Cabrera Cabrera, 2016). Así las cosas, esto ha llevado a que se proponga la modificación de la redacción de la norma en el sentido de que el ámbito de aplicación del EP de seguros aplique a todo tipo de agentes sin importar si es independiente o no, según los lineamientos de parágrafo 6 del artículo 5 de las plantillas tributarias (Huisman y Schuch, 2014).
Sobre este último punto, es preciso presentar la siguiente cita, la que ilustra directamente lo señalado con anterioridad:

Sin embargo, algunos países consideran que la disposición se debe ampliar para permitir el gravamen aun cuando la representación esté a cargo de un agente independiente. Adoptan ese criterio debido a la naturaleza del negocio de los seguros, al hecho de que los riesgos se localizan en el país que reclama la jurisdicción impositiva y a la facilidad con que algunas personas, trabajando a tiempo parcial, representan a compañías de seguros "a título independiente", lo que hace difícil distinguir entre agentes de seguros dependientes e independientes. Otros países consideran que no hay razón para tratar a las empresas de seguros de modo diferente a actividades como la venta de bienes tangibles (Comentarios al artículo 5, párrafo 29 Modelo de la ONU).

De igual manera, es palpable la preocupación de los Estados en vías de desarrollo en lo que respecta a la dificultad de poder atribuir beneficios a los agentes de seguros cuando existen varios de ellos realizando labores aseguradoras en el territorio. Sobre este punto, puede leerse lo siguiente:

También señalan la dificultad de determinar el monto total de las actividades realizadas cuando los seguros están a cargo de varios agentes independientes en el mismo país. Habida cuenta de esa diferencia de criterios, el trato reservado a los agentes independientes se decide en negociaciones bilaterales, que pueden tener en cuenta los métodos utilizados para la venta de los seguros y otras características del sector de los segu- 
ros en los países pertinentes (Comentarios al artículo 5, párrafo 29 Modelo de la ONU).

\section{El EP de seguros en el Derecho doméstico colombiano}

A raíz de la expedición de la Ley 1607 de 26 de diciembre de 2012, el Derecho Tributario colombiano adoptó el concepto del EP como mecanismo para determinar la imposición de las extensiones comerciales e industrias con naturaleza de permanencia de los no residentes fiscales. En términos generales, la definición interna del EP está basada principalmente en la definición prevista en el Modelo de la OCDE, acogiendo formas de EP como la regla base o la agencia.

Empero, en lo que concierne a nuestro objeto de estudio, es fundamental hacer hincapié en que nuestro precepto doméstico se caracteriza por la falta de alusión expresa al EP de seguros. En consecuencia, no es posible encontrar referencia alguna al EP de seguros ni en el Estatuto Tributario ni en los decretos reglamentarios respectivos. En conclusión y, de manera curiosa, la norma colombiana carece de los supuestos de la actividad aseguradora.

Basados en lo señalado, es de sana lógica colegir que la carencia del señalamiento expreso del EP de seguros lleva a que solamente podrán ser agentes de seguros aquellas personas que cumplan con los umbrales previstos en la cláusula de agencia doméstica. Así las cosas, la regla doméstica del EP personal establece:

También se entenderá que existe establecimiento permanente en el país, cuando una persona, distinta de un agente independien- te, actúe por cuenta de una empresa extranjera, y tenga o ejerza habitualmente en el territorio nacional poderes que la faculten para concluir actos o contratos que sean vinculantes para la empresa. Se considerará que esa empresa extranjera tiene un establecimiento permanente en el país respecto de las actividades que dicha persona realice para la empresa extranjera, a menos que las actividades de esa persona se limiten a las mencionadas en el parágrafo segundo de este artículo.

Parágrafo 1. No se entiende que una empresa tiene un establecimiento permanente en Colombia por el simple hecho de que realice sus actividades en el país a través de un corredor o de cualquier otro agente independiente, siempre que dichas personas actúen dentro del giro ordinario de su actividad.

No obstante, cuando el agente independiente realice todas o casi todas sus actividades por cuenta de tal empresa, y entre esa empresa y el agente se establezcan, pacten o impongan condiciones respecto de sus relaciones comerciales y financieras que difieran de las que se habrían establecido o pactado entre empresas independientes, dicho agente no será considerado como agente independiente para efectos de este parágrafo (Artículo 20-1 del Estatuto Tributario)

Como puede verse, la regla colombiana resulta análoga a la prevista en el Modelo de la OCDE, con lo cual el agente debe reunir los elementos analizados previamente, pero no debe ser considerado como de estatus independiente. Frente a este último punto, al no existir en la legislación local alguna pauta que señale los criterios que deben ser considerados para determinar si un agente es 
independiente, debemos remitirnos a los criterios de los comentarios antes referidos. Sin embargo, se puede evidenciar que, en caso de que el agente realice todas o casi todas sus labores a favor de una entidad imponiéndose condiciones que no se hubiesen presentado entre empresas independientes, dicho agente perderá su carácter de independiente. Frente a este último punto, puede evidenciarse cómo esta exclusión de independencia fue importada del Modelo ONU, aunque debe señalarse que también ha sido recientemente acogida por la OCDE como un nuevo criterio de la regla de agencia a través del instrumento multilateral del plan BEPS (Base Erosion and Profit Shifting).

Sin embargo, independientemente de si el agente local configura o no un EP, debe hacerse énfasis en que el desarrollo de una actividad aseguradora dentro de los límites territoriales del país constituirá un ingreso gravable en Colombia para el beneficiario en la medida en que el artículo 24 del Estatuto Tributario señala que se consideran ingresos de fuente nacional los derivados de la realización de actividades comerciales dentro del país.

Por último, consideramos importante poner de presente que la reciente reforma tributaria implementada en Colombia (Ley 1819 de 2016) introdujo una regla fiscal en virtud de la cual las oficinas de entidades foráneas dedicadas al negocio asegurador no son consideradas como EP en el país. Al respecto, puede leerse que

PARÁGRAFO 3. Las oficinas de representación de sociedades reaseguradoras del exterior no se consideran un establecimiento permanente (Artículo 20-1 del Estatuto Tributario).

\section{El EP de seguros en la red de tratados tributarios de Colombia}

A partir del año 2005, Colombia abandona su reticencia histórica de celebrar tratados de índole tributaria e inicia una política pública clara de procurar tener una red de CDI más extensa con la cual se pueda atraer mayor inversión extranjera, tanto directa como de portafolio (Quiñones, 2012; Mosquera, 2008).

A pesar de que el prototipo guía de negociación de Colombia ha sido preponderantemente el Modelo de la OCDE, es preciso hacer mención de que en ciertos CDI se han utilizado previsiones propias del Modelo de la ONU. Sentado lo anterior, es posible encontrar preceptos del EP de seguros en los tratados con Chile ${ }^{4}$, Corea del $\mathrm{Sur}^{5}$, India ${ }^{6}$, México $^{7}$, Portugal ${ }^{8}$ y República Checa 9 .

${ }^{4}$ Artículo 5 (7) del CDI Colombia-Chile.

${ }^{5}$ Artículo 5 (7) del CDI Colombia-Corea.

${ }^{6}$ Artículo 5 (7) del CDI Colombia-India.

${ }^{7}$ Artículo 5 (7) del CDI Colombia-México.

${ }^{8}$ Artículo 5 (7) del CDI Colombia-Portugal.

${ }^{9}$ Artículo 5 (7) del CDI Colombia-República Checa. 
Asimismo, es fundamental hacer énfasis en el hecho de que el EP de seguros incluido en los CDI colombianos referidos en el párrafo anterior son una fiel copia de la disposición del Modelo de la ONU. De tal suerte que los aspectos analizados previamente son plenamente aplicables a los tratados impositivos colombianos.

\section{Aplicación del EP de seguros en Colombia}

\section{A. La intermediación de seguros en el Derecho colombiano}

En lo que respecta a la legislación comercial, existe en el Código de Comercio un contrato típico mercantil denominado corretaje de seguros, dentro del capítulo del corretaje comercial. Dicho lo anterior, la norma dispone lo que se presenta a continuación:

ARTíCULO 1347. CORREDORES DE SEGUROS. Son corredores de seguros las empresas constituidas o que se constituyan como sociedades comerciales, colectivas o de responsabilidad limitada, cuyo objeto social sea exclusivamente ofrecer seguros, promover su celebración y obtener su renovación a título de intermediarios entre el asegurado y el asegurador.

En concordancia con la norma anterior, el artículo 2.30.1.1.2 del Decreto 2555 de 2010 (antiguo Artículo 2 del Decreto 2605 de 1993), señala que

La actividad de intermediación de seguros y reaseguros está reservada a las sociedades corredoras de seguros, a las sociedades corredoras de reaseguros, a las agencias colocadoras de seguros y a los agentes colocadores de pólizas de seguro, de acuerdo con su especialidad. La actividad de los intermediarios de seguros y reaseguros no inhabilita a las entidades aseguradoras para aceptar y ceder riesgos directamente, sin intervención de los intermediarios.

De la forma en que puede evidenciarse, es preciso señalar que se trata de un contrato cuya regulación se encuentra en el título del corretaje, lo que implica, por disposición expresa del artículo 1340 de dicho estatuto mercantil, que no se trata de ninguna forma de mandato o representación, ya sea directa $\mathrm{o}$ indirecta. $\mathrm{Al}$ respecto, léase que

ARTículO 1340. CORREDORES. Se llama corredor a la persona que, por su especial conocimiento de los mercados, se ocupa como agente intermediario en la tarea de poner en relación a dos o más personas, con el fin de que celebren un negocio comercial, sin estar vinculado a las partes por relaciones de colaboración, dependencia, mandato o representación.

Por lo tanto, se trata llanamente de una relación contractual en virtud de la cual una persona llamada corredor actúa como intermediario entre dos o más personas, para que estas celebren un negocio jurídico y que, en caso de concretarse dicho negocio, el corredor tiene derecho a una remuneración por su intermediación. Asimismo, es una actividad que se encuentra sujeta a disposiciones especiales del Estatuto Orgánico Financiero y al estricto cumplimiento de múltiples reglas contables, financieras, prudenciales, entre otras, que no son reseñadas porque exceden el propósito del texto. 


\section{B. Falta de norma doméstica del EP de seguros}

El primer punto álgido frente a la aplicación de la regla del EP de seguros para el caso colombiano resulta en el interrogante frente a si es posible aplicar la regla de seguros aun cuando la norma doméstica no prevé esta hipótesis. Al respecto, debe señalarse que, en virtud del sistema jurídico colombiano, los tratados internacionales, incluidos naturalmente los de naturaleza tributaria, deben ser ratificados mediante ley de la república. Por lo tanto, hay una incorporación normativa del texto aprobatorio del tratado, la cual tiene plena vigencia en nuestro ordenamiento jurídico-tributario.

Lo señalado en el párrafo anterior nos permite poner de presente un conflicto hermenéutico entre dos disposiciones legales de similar naturaleza. Así las cosas, para solventar esta dificultad se requiere acudir a las normas generales de interpretación de las normas jurídicas. Sentado lo anterior, la Ley 57 de 1887 en su artículo 5 señala que cuando haya una incompatibilidad entre dos normas de similar jerarquía se preferirá aquella que tenga carácter especial. Por lo tanto, es pertinente concluir que el tratado tributario goza de una connotación de especialidad y, por lo tanto, prevalece sobre las normas internas del Estatuto Tributario generando que pueda existir una hipótesis de EP de seguros en aquellos CDI que así lo prevean.

\section{Requisitos para la existencia de un agente de seguros desde la óptica doméstica}

Desde un punto de vista del Derecho Tributario doméstico, como se señaló, para que pueda activarse un EP de seguros es menester la existencia de un agente dependiente que obligue legalmente a la empresa no residente. Ahora, bajo el régimen de representación colombiano, basado a su vez en la tradición romano-germánica, la hipótesis anterior solo ocurre en los casos del mandato con representación o contemplatio domini. Entablado lo precedente, no sería posible la existencia de agentes de seguros en eventos en los cuales se hayan celebrados contratos de mandato sin representación, como, por ejemplo, un contrato de comisión. Esto se desprende de que el artículo 20-1 del Estatuto Tributario excluye del EP de agencia a los corredores.

\section{Conclusiones}

1. El establecimiento permanente de seguros es un supuesto especial originario del Modelo de Convenio de la ONU, el cual procura ampliar el espectro de radio de la tributación in situ. Asimismo, son dos sus elementos constitutivos: a) un agente dependiente, según las normas del artículo 5(5) del Modelo de la OCDE, y b) una actividad de seguros.

2. A pesar de que la norma doméstica del EP se caracteriza por la carencia de la definición del EP de seguros, el que Colombia en algunos de sus convenios bilaterales tributarios haya incluido este tipo de provisiones, implica concluir que es posible la aplicación de esta forma especial de EP debido a la especialidad de las leyes aprobatorias de los tratados fiscales.

3. Basados en las normas del Código de Comercio, al ser la intermediación de seguros una típica relación de corretaje, será un 
factor que caracterice al agente como uno de naturaleza independiente.

4. Bajos las normas de los CDI, es imperativo que el agente de seguros no sea considerado independiente, haciendo que en la práctica sea poco probable la existencia de un EP de seguros, dada la naturaleza per se de independencia de estos últimos. Por lo tanto, consideramos acertada la postura de no atar el EP de seguros a la independencia del agente. 


\section{Bibliografía}

Cabrera Cabrera, O. (2016). El Establecimiento Permanente: especial énfasis en la Cláusula de agencia. Bogotá, Colombia: Universidad Externado de Colombia.

Cabrera Cabrera, O. (2017). “Aspectos tributarios de las plataformas digitales en Colombia: actualidad y retos”. Revista de Derecho Fiscal, (X), 85-101.

Dunahoo, C A. (2008). "Source country taxation of foreign corporations: Evolving permanent establishment Concepts". Taxes-The Tax Magazine.

Fuentes Hernandez, D. (2014). "Agents of an Idependenpent Character under Article 5(6) of the OECD Model Convention”. Lang et al. (eds.). Dependent Agents as Permanent Establishment. Viena, Austria: Linde.

García Prats, A. (1996). El Establecimiento Permanente: Análisis jurídico- tributario internacional de la imposición societaria. Madrid, España: Tecnos.

Goblit, Gabriel y Vaquero, Fernando M. (2011). Aspectos Internacionales de la Tributación Argentina: Estudio Teórico-Práctico. Buenos Aires, Argentina: La Ley.

Huisman, E. y Schuch, J. (2014). "The Dependent Agent PE under Article 5(5), (6) and (7) of the UN Model Convention”. Lang et al. (eds.). Dependent Agents as Permanent Establishment, Viena, Austria: Linde.

Quiñones Cruz, N. (2012). "Colombia Report". Lang et al. (eds.) The Impact of the OECD and UN Model Conventions on Bilateral Tax Treaties. Cambridge, Reino Unido: Cambridge Tax Law Series.

Mosquera Valderrama, I. (2008). “The International Tax Treaty Policy of Colombia, en Bulletin for International Taxation". Bulletin for International Taxation, vol. 67 Issue N 4.

Reimer, E. (2016). "Permanent Establishments in the OECD Model Tax Convention”. Reimer, Schmid and Orell (eds.). Permanent Establishments: A Domestic Taxation, Bilateral Tax Treaty and OECD Perspective. Frederick, Estados Unidos: Wolters Kluwer Law \& Business.

Skaar, A. (1991). Permanent Establishment: Erosion of a Tax Treaty Principle. Boston, Estados Unidos: Kluwer Law and Taxation Publishers.

Sasseville, J. y Skaar, A. (2009). "General Report". Is there a Permanent Establishment? 63 ${ }^{\text {er }}$ Congress of the International Fiscal Association. Vancouver, Canada: Cahiers de Droit Fiscal International by the International Fiscal Association.

Tillinghast, D R. (1993). "The choice of issues to be submitted to arbitration under income tax conventions". Alpert y Van Raad (eds.). Essays on International Taxation in honor of Sydney I Roberts. Deventer, United States: Kluwer Law and Taxation Publishers.

Title, M. (2007). Permanent Establishment in the United States: A view through Article V of the U.S. - Canada Tax Treaty. Toronto, Canada: Vandeplas Publishing.

Vogel, K. (2011). On Double Taxation Conventions, A Commentary to the O.E.C.D, UN, and US Model Conventions for the Avoidance of Double Taxation on Income and Capital with Reference to German Treaty Practice. Boston, United States: Kluwer Law International. 